Iraqi Journal of Statistical Science (8) 2005

P.P. $[15-21]$

\title{
HIGH RESOLUTION IMAGE CLASSIFICATION
}

Wasfi Taher Saalih*

E-mail wasfit@yahoo.com

- Statistics Department - College of Administration \& Economics - Salahaddin University

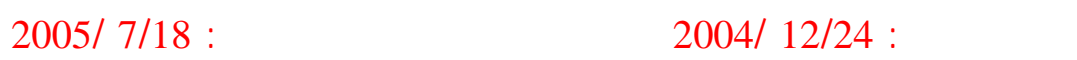




\section{ABSTRACT}

Classification is an important field with many applications. In particular, the classification of digital imagery has important applications in the mapping community. In this paper the comparison of three different classification methods on LANDSAT imagery of Erbil City - IRAQ: neural networks, nearest-neighbor, and discriminant analysis are made.

Out of the three approaches, k-nearest neighbors performed the best; next in accuracy was neural networks, and then discriminant analysis.

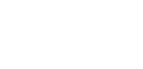

يعد التصنف جقلا مهما في عدة نواح قطبية. وعلى وجه الخصوص، لت ـصنف الصور الرقمية دور مهم في محط الخرائ. في هذا البحث تمت مقارنة ثلاث طررائق

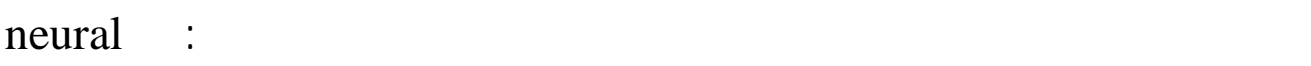
networks, nearest-neighbor, الثلاث كان ـت nearest-neighbor الاف مضل وتليه L Leural networks ولخي -را .discriminant analysis

\section{INTRODUCTION}

Remotely sensed imagery can be used for many applications, including reconnaissance, creation of mapping products for military and civil applications, assessment of environmental damage, land use monitoring, radiation monitoring, urban planning, growth regulation, and soil evaluation. Classification of this imagery is usually an essential part of these applications. While in the past this imagery usually has been of moderate resolution (e.g. LANDSAT 30-meter pixels and SPOT 20-meter pixels), more recent imagery 
has been of much higher resolution (e.g. 4-meter, 2-meter, 1-meter pixels, or less than 1-meter pixels). In particular, one of the authors has been investigating computational issues surrounding large, multi-band digital imagery sets of $10^{\wedge} 6-10^{\wedge} 8$ pixels and $4-256$ separate bands per pixel. Previous work has centered on the use of spatial information for compression of the imagery without significant information loss [Shine 2000, 2001, and 2002]. In this paper, the authors investigate the classification of uncompressed moderate-resolution and high-resolution imagery.

Classifying at high resolutions such as 1-meter pixels or less than 1-meter pixels offers advantages over classification at lower resolutions such as 20-meter or 30-meter imagery. For many applications the extra resolution is needed for precision. Classification at high resolutions also greatly reduces the problem of mixed pixels (when a pixel contains more than one type of land cover, e.g. forest and road). The big disadvantage of classifying high-resolution imagery is the computational issue. Wegman has examined the issues surrounding large data set computations in detail [Wegman, 2001].

In this paper three classification methods are taken under consideration and compare a both the accuracy of the method and the size limitations of existing computer software implementations.

\section{DATA}

The data set was 1-meter, 4-band multispectral imagery data of Erbil city - Iraq. Since there are published results testing 4 of the 5 classification methods, this data set served as an excellent base to test software and as a benchmark for accuracy.

The imagery was collected by Joint Humanitarian Information Center (JHIC) in Erbil IRAQ. The full data set was approximately 2,000 rows by 2,000 columns for a total number of $4 \times 10^{\wedge} 6$ pixels per band; there were four bands, at 450, 550,650 and $800 \mathrm{~nm}$ (blue, green, red and near infrared) respectively. Several hundred of the pixels were classified by analysts to use as training and test points in 
the classification analysis. For each pixel there are four image variables (one for each band) and a class variable with six values: road, manmade, deciduous trees, pine trees, water, and field/grass.

Figure 1 shows a view of the imagery of Erbil City Iraq.

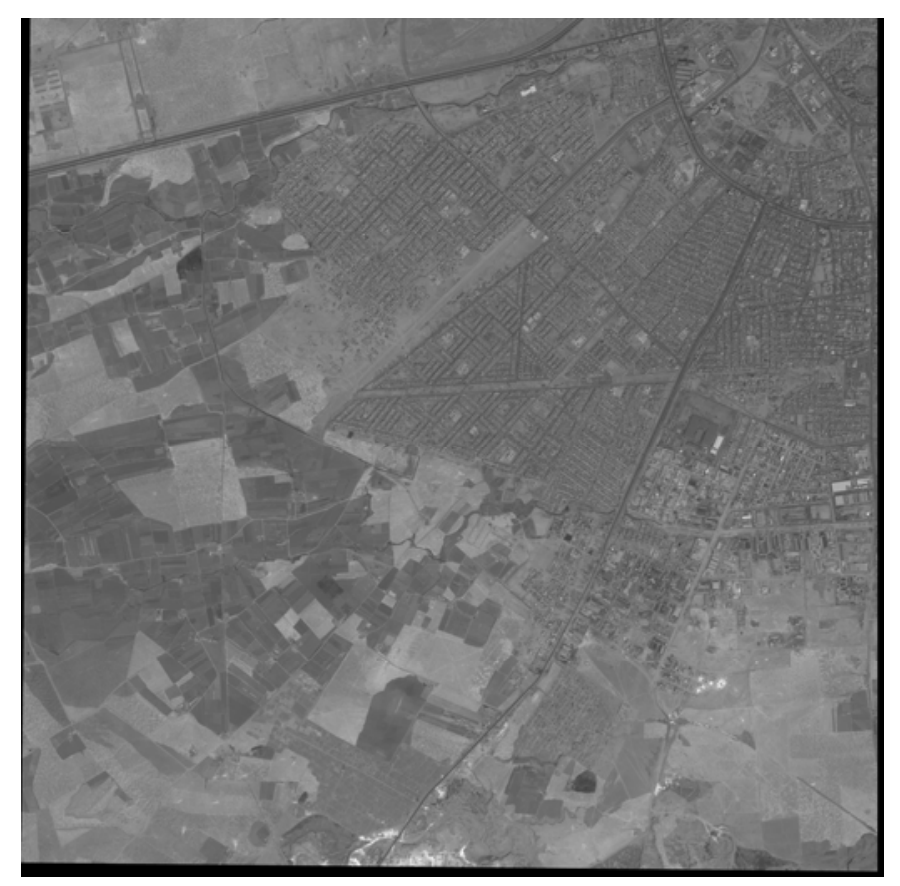

Figure 1, Imagery of Erbil city - IRAQ

\section{METHODOLOGY}

As mentioned in the introduction, three separate classification approaches were tested on the imagery data sets. A brief description of each of the five methods and the available software for those methods follows.

\section{Neural Networks}

Neural networks originally developed from models loosely based on biological neurons. They can be represented statistically as two or 
more nested functions: for example, $y=f(W), W=g(X)$. For imagery data $\mathrm{X}$ would be the image variable and $\mathrm{y}$ would be the class variable. Training points are used to correct the parameters of $f$ based on error functions, and then the revised $\mathrm{f}$ parameters are used to correct the parameters of g. There is an abundance of software available to perform basic gradient descent neural network classification. A neural network library for S-Plus developed by Ripley was used for this paper. Other code exists in Matlab, C and FORTRAN, and various commercial packages exist.

\section{Discriminant Analysis}

Discriminant analysis dates back to the 1930s and R.A. Fisher [Anderson, T.W., 1984]. Training values are used to compute means and covariances for the classes, and then new data observations are classified by determining which class is "closest" in distance to the point (usually a Gaussian, L2-norm type distance such as Mahalanobis distance). Covariances may assume the same for all classes or different for each class. There is software available in SAS and S Plus among other packages, and both SAS and S Plus were used for this paper.

\section{K-Nearest Neighbors}

The k-nearest neighbor approach is quite simple [Anderson, T.W., 1984]. For each data point, the $\mathrm{k}$ neighboring training points with known class values which are closest in distance to the point are chosen. The class which occurs most often in the neighbors is assigned to the new point. The approach works best when the data has spatial properties, which is usually the case with imagery. The approach is very simple computationally. A library function for SPlus by Ripley was used for this paper.

\section{ANALYSIS AND RESULTS}


Each of the three methods was tested using both sets of imagery. Two separate comparisons were performed; first, a comparison of classification accuracy, and second, a comparison of computational limitations for a particular software implementation. All computations were performed on a Pentium III single processor CPU with 700MHz speed and $256 \mathrm{MB}$ RAM.

\section{Neural Networks}

Ripley's “nnet” library package (added to S-Plus) achieved 85\% accuracy on the Erbil City data. Expanding the data set to $34 \mathrm{~K}$ observations $\left(\sim 10^{\wedge} 4\right)$ lengthened computation time to 600 seconds, and expanding the data set to $136 \mathrm{~K} * \sim 10^{\wedge} 5$ ) caused the program to freeze. Again, compression would be necessary to classify a full high-resolution image.

\section{Discriminant Analysis}

Both SAS (PROC DISCRIM) and S-Plus(lda) programs achieved 83\% accuracy on the Erbil City. There was a slight difference between the two approaches, with S-Plus outperforming SAS by approximately $0.1 \%$, which is probably due to a slight difference in the computation of the covariance matrices (SAS uses a pooled matrix, S-Plus does not). S-Plus again froze at $\sim 10 \wedge 5$ data points. SAS did not freeze up to $10^{\wedge} 6$ points so compression is not a big issue for this approach, but the based on the accuracy results, discriminant analysis is not an optimal classifier.

\section{K-Nearest Neighbors}

Ripley's "knn” library (added to S-Plus) achieved an 88\% accuracy on the A.P. Hill data. Again the computational limit was approximately $10^{\wedge} 5$ points.

\section{CONCLUSIONS}

Of the three approaches, k-nearest neighbors performs the best, achieving an accuracy of around $88 \%$. Next in accuracy were 
neural networks at $85 \%$, and then discriminant analysis at $83 \%$. All of the methods had computational limits significantly smaller than that of a full high-resolution imagery data set except the poorest performer, discriminant analysis. While some of the computational limits are the result of the S-Plus memory manager and can be increased by the use of $\mathrm{C}$ or FORTRAN code or open source R code (which has a different memory manager than S-Plus) it is still evident that classification of a full high-resolution image is a major computational challenge, and this underscores the importance of compression approaches such as those discussed in the introduction.

\section{REFERENCES}

Anderson, T.W., 1984, "An Introduction to Multivariate Statistical Analysis", John Wiley \& Sons $2^{\text {nd }}$ edition.

Michie,D., Spiegelhalter, D.J., and Taylor, C.C., 1994, "Machine Learning, Neural and Statistical Classification”, Ellis Horwood.

Shine, J.A (2000), "Mapping and Modeling 1-Meter Multispectral Imagery Data", 2000 Proceedings of the American Statistical Association, Alexandria, VA: American Statistical Association.

Shine, J.A. (2001), "Compression and Analysis of Very Large Imagery Data Sets Using Spatial Statistics”, Proceedings of the $33^{\text {rd }}$ Interface Symposium, Costa Mesa, CA, June 2001.

Shine, J.A., and Carr, D.B. (2002), "Relationships between Land Cover and Spatial Statistical Compression Capabilities in HighResolution Imagery", Proceedings of the $34^{\text {th }}$ Interface Symposium, Montreal, CA, April 2002.

Wegman, E.J. (2001), "Data Mining and Visualization: Some Strategies”, George Mason University Center for Computational Statistics, Technical Report 166, July, 2001. 